\title{
Du corps à l'image. Repenser les performances culturelles en Océanie à l'ère de la mondialisation
}

The body and its image: reinventing cultural performances in Oceania in the era of globalization

\section{Aurélie Condevaux, Géraldine Le Roux et Laura Schuft}

\section{(2) OpenEdition}

\section{Journals}

Édition électronique

URL : http://journals.openedition.org/jso/7591

DOI : $10.4000 /$ jso.7591

ISSN : $1760-7256$

Éditeur

Société des océanistes

Édition imprimée

Date de publication : 31 décembre 2016

Pagination : $5-20$

ISSN : 0300-953x

Référence électronique

Aurélie Condevaux, Géraldine Le Roux et Laura Schuft, « Du corps à l'image. Repenser les

performances culturelles en Océanie à l'ère de la mondialisation », Journal de la Société des Océanistes [En ligne], 142-143 | 2016, mis en ligne le 15 décembre 2018, consulté le 24 septembre 2020. URL : http://journals.openedition.org/jso/7591 ; DOI : https://doi.org/10.4000/jso.7591 


\section{Du corps à l'image. La réinvention des performances culturelles en Océanie à l'ère de la mondialisation}

par

Aurélie CONDEVAUX*, Géraldine LE ROUX ${ }^{* *}$ et Laura SCHUFT ${ }^{* * *}$

Dans une ère de mondialisation et de nouvelles technologies de communication, et dans le fil d'histoires profondément coloniales, comment se pensent et s'organisent les performances culturelles en Océanie aujourd'hui ? Que révèlent ou véhiculent les mises en scène et en images des cultures océaniennes? Quels cadres interprétatifs nous permettent de saisir les revendications sociales et politiques et les processus culturels qui se déroulent à l'arrière des ou sur les scènes ? C'est à travers cet ensemble de questions que ce dossier collectif entend renouveler le regard sur un objet d'étude ancien : les performances à la fois corporelles et culturelles en Océanie.

Dans la lignée de travaux anthropologiques récents, nous désignerons par le terme de "performances" des pratiques variées, en particulier des chants et des danses (Glowczewski et Henry, 2007), du théâtre (Préaud, 2007), voire des séquences de gestes et de paroles qui accompagnent la présentation d'œuvres peintes. Notons que certaines de ces performances réalisées dans un cadre muséal sont élaborées comme une réponse à une histoire de l'art occidental et à un cadre interprétatif pensé comme restrictif (Le Roux, 2007). Les performances peuvent donc être décrites comme des "développements intentionnels de compétence expressive devant un public" (Castellani, 2007 : 74) et qui sont distingués du quotidien à l'aide de divers moyens, comme la définition d'un espace, d'une "scène", un choix de costumes ou d'autres éléments qui tranchent avec l'ordinaire.

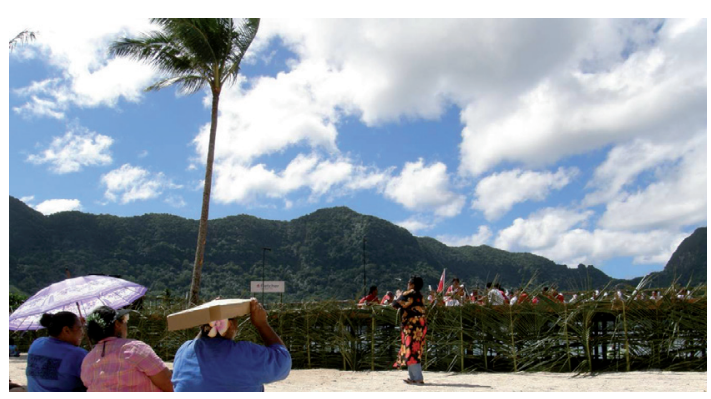

Рното 1. - Dixième Festival des Arts du Pacifique (C Le Roux, 2008, Pago Pago, Samoa américaines)

Dans ce sens, et contrairement à la définition proposée par A. Kaeppler (2002), les performances excluent le "rituel ", entendu lui comme séquence d'actes formels dont la signification échappe aux performeurs. Les performances dont il est question dans ce numéro ont un public humain (alors que, dans le cas des rituels, le public peut être divin) et elles véhiculent un message plus ou moins " codé " (Kaeppler, 2010b), dont les contributeurs de ce numéro discutent l'élaboration ou la réception dans un contexte local et global. De ce fait, la question de l'audience est essentielle et l'on peut distinguer, comme le fait A. Kaeppler (2010b), les membres du public qui ont les compétences pour décoder ces messages dans toute leur complexité de ceux qui en sont dépourvus, et qui sont de ce fait de simples "spectateurs". Cette dernière situation est fréquente lorsque les performances

NDLR. - Cette introduction est disponible en anglais sur jso.revues.org et sur cairn international/This presentation is avalaible online in English, see jso.revues.org and http://www.cairn.info/revue-journal-de-la-societe-des-oceanistes-2016.htm.

*Université Paris 1 Panthéon-Sorbonne, EIREst, aurelie.condevaux@univ-paris1.fr

** Université de Bretagne occidentale, CRBC, geraldine.leroux@univ-brest.fr

*** Université Côte d'Azur, LAMHEss, laura.schuft@unice.fr 
sont "décontextualisées » et produites dans le cadre de festivals régionaux et/ou internationaux. Les articles rassemblés dans ce numéro présentent des situations caractéristiques de ces différents cas de figure. Ils mettent également en lumière ce qu'implique le passage du rituel à la performance, ou les mécanismes de résistance à un tel passage.

Les performances corporelles et la diffusion de leurs images sont au cœur de processus sociaux majeurs. Elles jouent par exemple un rôle central dans l'élaboration des identités sociales et culturelles. La formule utilisée par $S$. Tcherkézoff pour définir la société, à savoir la manière dont les acteurs « disent et mettent en pratique une appartenance à un même tout, ce dernier étant plus restreint que l'ensemble formé par le genre humain " (Tcherkézoff, 1997 : 310), semble pouvoir être réutilisée pour définir les «identités ». S'intéresser aux identités, c'est s'intéresser aux processus d'identification par lesquels celles-ci « are subjectively defined, described, and experienced by individuals» (Morton Lee, 2003 : 3), étant entendu que ces processus de définition ou négociation sont indissociables des interactions sociales dans lesquelles ils s'inscrivent (Goffman, 1973). De plus, les constructions identitaires opèrent par le biais d'une sélection de traits culturels dont les acteurs se saisissent pour en faire des marqueurs d'appartenance (d'un point de vue interne) ou d'assignation (d'un point de vue externe). Du fait de ces liens étroits avec les processus de construction des identités culturelles, nous parlerons de "performance culturelle» pour désigner l'objet des différentes contributions à ce numéro.

De nombreux travaux ont noté la place centrale occupée par le corps dans ces processus d'identification (Le Breton, 2016). Dans son travail sur le genre, J. Butler (2006) montre comment les pratiques corporelles, dans leur répétition, participent fortement à réifier les identités, les caractéristiques et les différences sociales. L'inscription de ces dernières dans - et leur expression par - le corps contribuent à leur donner une apparence " naturelle ». La question du corps et de ses rapports aux structures sociales (Bourdieu, 2000) amène inévitablement à la question des relations de pouvoir. L'étude de performances dans lesquelles le corps joue un rôle central permet de saisir la manière dont se reproduisent ou se négocient des rapports sociaux de pouvoir ou des tensions politiques. En tant que manifestations publiques, visibles et qui ont un «impact émotionnel immédiat " (Alexeyeff, 2009 : 13), les performances culturelles sont en outre un emblème aisément transposable dans différents contextes au sein desquels leur contenu et leurs significations peuvent varier. Il y a un parallèle entre la mobilité des corps et celle des identités construites performativement, lesquelles peuvent prendre des formes et des sens différents selon les contextes de mise en ouvre, de diffusion et de réception de la performance. Le haka par exemple, selon D. Murray (2000), peut servir à la fois d'identification pan māori, d'identification «tribale " (au niveau du iwi ou hāpu, voir ci-dessous) ou familiale (whänau). Cette diversité des significations opère également à une échelle locale. G. Petersen (1992) examine comment une danse peut véhiculer des messages multiples, qui portent sur les relations que les " groupes " ou " communautés " - en l'occurrence de Pohnpei - entretiennent entre eux et/ou avec l'extérieur. Cette multiplicité des significations est susceptible d'être décuplée par l'utilisation des nouveaux médias de communication. La diffusion de l'image des corps et des performances à des publics nouveaux et, de plus en plus larges, par le biais des technologies de communication, multiplie l'impact de ce que dit le corps sur les identités ou différences sociales et culturelles (Schuft, 2012).

L'un des enjeux du numéro est de saisir ce qui se passe dans cette translation "du corps " à l'" image ". Les nouveaux moyens de communication et la circulation des performances questionnent-ils la place du corps dans la définition des relations de pouvoir et des identités? Exacerbent-ils au contraire l'expression de ces dernières, réduisant les performances à des "rituals of identity" (Kaeppler, 2002) au détriment de leurs autres enjeux sociaux et culturels ?

Avant de présenter les contributions du dossier et les manières dont elles renouvellent le regard sur les performances culturelles en Océanie, nous proposons d'abord une synthèse de la vaste littérature anthropologique sur ces dernières, dont les objets sont majoritairement des performances chorégraphiques et musicales. Nous discutons, d'une part, les manières dont les performances, en tant qu'objet d'étude, permettent d'observer les transformations sociétales et, d'autre part, quels sont les cadres analytiques mis en place pour leur étude.

\section{Les performances culturelles : miroirs de la société ou forces génératives du changement social}

Les travaux sur les pratiques musicales et chorégraphiques en Océanie abondent. Une compilation exhaustive des recherches faites sur ce sujet semble impossible: parcourir la bibliographie annotée de M. McLean (1977) suffit à s'en convaincre. Cette littérature est faite de travaux de chercheurs occidentaux et d'auteurs océaniens - chorégraphes, compositeurs, performeurs - qui ont fréquemment partagé leurs connaissances, en tant qu'experts reconnus de ces pratiques, sous forme de publications, en particulier sur des contextes où les pratiques chorégraphiques ont acquis un statut emblématique fort (Armstrong et Ngata, 2002 ; Gardiner, 2005, Huata, 2000 ; Kāretu, 1993). Au-delà 
des publications d'ethnomusicologues (Linkels et Linkels, 1999 ; McLean, 1999) qui s'attachent principalement à décrire les différentes catégories et genres de musique et de danse, archipel par archipel, donnent des indications précises sur les techniques corporelles, les types de mouvement et détaillent parfois des compositions (paroles, musique, gestuelle), nous pouvons retracer dans les grandes lignes la manière dont l'étude des pratiques musicales et chorégraphiques a contribué aux domaines de recherche qui sont au cœur de l'anthropologie océanienne.

Le travail ethnographique sur ces pratiques qui prend son essor au début du $\mathrm{Xx}^{\mathrm{e}}$ siècle (Best, [1925] 1976 ; Burrows, 1945; Krämer, 1995) - a notamment permis de mettre en évidence les termes permettant d'exprimer les idées relatives à la beauté, les manières de manifester l'appréciation esthétique et la variété des émotions impliquées ou générées par la performance (Konishi, 1999 ; Kaeppler, 1993 ; Tamisari, 2000). Mais les analyses ont surtout mis en exergue l'articulation de ces pratiques aux structures sociales, que ce soit les systèmes religieux, les relations de parenté, les transactions économiques ou l'organisation politique, autant de domaines sociaux que nous proposons ici d'évoquer point par point.

En ce qui concerne la religion, les travaux s'intéressent aussi bien à la place des pratiques chorégraphiques et musicales dans les cultes religieux préchrétiens qu'aux dynamiques générées par le contact avec les Occidentaux et la christianisation. À Chuuk (anciennement Truk), une île de Micronésie, des spécialistes rituels (ritual specialists) étaient chargés, par des récitations précises, de guider le pouvoir spirituel (manaman) (Diettrich, Moulin et Webb, 2011: 83). Lors de possessions rituelles, les esprits des ancêtres pouvaient également intervenir dans le monde des vivants, notamment pour révéler de nouvelles chansons. En Papouasie Nouvelle-Guinée, danses et musiques (en particulier les flûtes sacrées) manifestent fréquemment la présence des esprits ancestraux, que ce soit par exemple dans les Hautes Terres (Diettrich, Moulin et Webb, 2011 : 88-89) ou dans la province de Madang (Smidt et Eoe, 1999). Bien que la matérialisation de la présence des esprits ancestraux ou mythiques dans les masques ait davantage retenu l'attention des ethnographes que les danses et musiques qui les accompagnent (Smidt et Eoe, 1999; Vienne, 1996), certaines analyses fines en ont été proposées. P. Wolffram (2011) par exemple montre, à partir de l'étude d'une cérémonie marquant la fin de funérailles chez les Lak de Nouvelle-Irlande, toutes les facettes de l'imbrication des pratiques chorégraphiques et musicales avec les représentations de l'environnement et du monde spirituel. Les esprits apparaissent comme la source de la création musicale, tandis que la qualité de la gestuelle et les types de mouvement exécutés confèrent aux danseurs des qualités généralement attribuées aux esprits, telles la légèreté ou la rapidité. Tout, dans les performances dansées des masques, témoigne de la coopération étroite entre les esprits et les hommes, la performance elle-même prenant d'ailleurs place dans un espace liminal se situant entre la maison des hommes et la forêt, l'espace des esprits.

En dépit des tentatives d'interdiction des missionnaires, les pratiques musicales et chorégraphiques anciennement déployées dans des contextes religieux ont parfois subsisté, tout en revêtant des significations nouvelles. La question du passage du sacré au séculier est notamment évoquée par W. Donner (1992: 74). A. Kaeppler (2002) a consacré également plusieurs publications à ce sujet. Elle défend l'idée que des séquences de sons et de mouvements qui trouvaient autrefois leur origine dans des espaces sacrés ont été transformés: de « travail » religieux (religious 'work), elles relèvent désormais de la "musique " et de la "danse ", servant de "marqueurs identitaires" ou " marqueur d'identité ethnique " pour reprendre deux expressions utilisées par l'auteur (2002: 8). C'est le cas $\mathrm{du}$ hula hawaiien qui est passé du domaine religieux à celui de l'affirmation identitaire (Balme, 2007 ; Kaeppler, 2002, 2010a, 2010b). Ce changement de sens (Kirshenblatt-Gimblett, 1998) et cette " sécularisation " des danses sont parfois liés de manière étroite aux dynamiques propres aux diasporas. Il en va ainsi de la danse bon, pratiquée par les Japonais et les descendants de Japonais vivant à Hawai' i, qui, dans certaines circonstances, tend à perdre son lien initial au bouddhisme (Van Zyle, 1988). Mais la participation à des festivals nationaux ou transnationaux peut être le lieu d'une réactualisation des anciennes significations, en l'occurrence religieuses. En dehors de ces processus de "sécularisation" se pose également la question de la place qui a été accordée aux danses et aux chants considérés comme "traditionnels" dans les Églises chrétiennes. À partir d'une analyse ethnohistorique des Églises protestantes de Polynésie, Y. Fer (2009) montre, par exemple, qu'après des périodes d'interdit ou de rejet, les danses polynésiennes ont pu être réappropriées dans l'« élaboration d'une identité trans-polynésienne " au sein de certaines congrégations religieuses.

Lexécution de performances musicales et chorégraphiques lors de grandes célébrations est en outre l'occasion d'activer les coopérations familiales, de montrer les réseaux de parenté et de susciter en même temps diverses transactions économiques. Pour les Yolngu d'Australie, les danses et les chants sont la propriété de groupes spécifiques de parents qui ont le droit et la responsabilité de les exécuter (Tamisari, 2000). Dans son analyse des relations clownesques des Murik de Papouasie Nouvelle-Guinée, K. Barlow (1992) observe également que l'exécution de certaines danses au moment des funérailles - en 
particulier des danses humoristiques ou provocatrices, mais également "sérieuses " - relève du devoir de certaines catégories de personnes, en l'occurrence ici des parents classificatoires ou fictifs appelés mwara. Ces performances s'inscrivent au sein des relations à plaisanterie qui permettent de faire face aux contradictions de la vie sociale et aux exigences antagonistes auxquelles sont soumis les individus.

L'exécution de chorégraphies ou de chansons peut requérir le paiement d'une compensation du fait des "droits de propriété " de l'auteur ou d'un groupe sur certaines compositions. Cela a particulièrement été montré en Papouasie Nouvelle-Guinée (Smidt et Eoe, 1999) et au Vanuatu (Stern, 2013). En Polynésie, l'idée de "propriété " est beaucoup moins forte ou ne s'exprime pas de la même manière (McLean, 1999 : 391). En effet, si le compositeur ou le chorégraphe est toujours reconnu pour son travail - qui fait souvent l'objet de compensations matérielles ou d'une reconnaissance sociale -, il ne s'agit pas de céder des droits sur cette composition contre rémunération. L'idée de mettre une œuvre en vente - au sens où celle-ci serait aliénée - fait peu de sens pour les acteurs (McLean, 1999 : 391). Plus largement, les danses peuvent être un vecteur de circulation des richesses, sans que la transaction ne se réduise ni à l'achat d'une composition/ chorégraphie, ni à la rémunération du travail effectué. Il peut s'agir de "dédommager le désir " suscité par l'émotion esthétique, comme le souligne M. Jeudy-Ballini (1999) dans le cas des Sulka de Nouvelle-Bretagne. Une logique similaire existe en Australie, chez les Yolngu, où le danseur virtuose se voit pressé par l'audience de donner des biens matériels ou de l'argent, une manière de s'assurer qu'il ne fera pas un usage excessif de son talent en humiliant les autres (Tamisari, 2000). À l'inverse la performance peut appeler le don, comme c'est le cas dans différents archipels de Polynésie. Aux îles Cook ou à Tonga par exemple, les prestations suscitent des gratifications diverses, d'objets (tissus d'écorce, colliers de fleurs, etc.) ou de billets, parfois collés à même la peau huilée ou déposés aux pieds des danseurs (Alexeyeff, 2009; Condevaux, 2010). Dans ces différents cas, on note une imbrication étroite entre l'émotion esthétique, les relations interpersonnelles et la circulation de biens matériels.

Les pratiques chorégraphiques sont en outre une porte d'entrée privilégiée pour étudier le politique. Pour la Papouasie Nouvelle-Guinée par exemple, on sait que les "festivals" (des cérémonies marquant un moment important du cycle de vie) font une place centrale aux pratiques musicales et chorégraphiques tout en offrant l'occasion aux big men de réaffirmer leur prestige social (Smidt et Eoe, 1999 ; Wolffram, 2011). La virtuosité dans la danse peut être à la fois une manifestation de la présence des ancêtres et une expression de la position d'autorité de certains individus (Tami- sari, 2000 : 281). Dans l'aire polynésienne également les connexions sont étroites entre danse, musique et structure politique. L'utilisation des récitations chantées pour célébrer l'accomplissement des chefs est bien connue dans cette région, comme à Hawaii i où elles célèbrent aussi parfois leur beauté et leurs capacités reproductrices (Sahlins, 1989 : 29-33). A Tonga également, danses et musiques (faiva) mobilisent souvent des textes qui font l'éloge des lignées de " rois » ou " chefs". Dans cet archipel de Polynésie occidentale, les danses sont une visualisation, dans leur structure et dans leur organisation mêmes, de la hiérarchie sociale, et leur préparation s'avère être un lieu d'apprentissage des statuts assignés dès la naissance (statuses ascribed by birth) (Kaeppler, 1993 : 58). En Micronésie, pratiques chorégraphiques et musicales sont aussi étroitement associées à l'affirmation et/ou à la reproduction des structures politiques hiérarchiques. À Pohnpei par exemple, l'un des États fédérés de Micronésie, organisé en cinq royaumes (chiefdoms) indépendants, les interactions politiques, que ce soient celles entre les différents royaumes ou avec l'administration coloniale, sont fortement valorisées. Des dance songs (koulin kablek) - encore produites lors d'événements sociaux importants - racontent l'histoire de ces interactions (Diettrich, Moulin et Webb, 2011 : 24).

Permettant de saisir l'organisation sociale dans son ensemble et, en particulier, les structures hiérarchiques, l'étude de la danse a été un point d'entrée pour aborder la question du changement social opérant sous l'effet de facteurs divers. Comme le rappelle K. Nero (1992) en introduction du numéro thématique de Pacific Studies: The Arts and Politics, le lien entre les productions esthétiques et le système politique ne doit pas être réduit à un " reflet " du second par les premières, ni au rôle d'instrument de légitimation du pouvoir que peuvent constituer musiques et danses. Au contraire, les performances sont souvent le lieu ou le moyen par lequel des messages subversifs peuvent être délivrés.

C'est dans cette même perspective que $\mathrm{K}$. Alexeyeff (2009) envisage les productions artistiques comme des forces génératives plus que comme des miroirs de la vie sociale. Selon l'auteur, les pratiques artistiques contribuent à façonner les cadres de l'action politique, économique ou sociale $(2009$ : 12). R. Henry $(2000 ; 2007)$ invite également à penser en ce sens. Elle souligne que le corps est le plus sûr moyen d'une agentivité : c'est dans la performance culturelle que peut se jouer la remise en cause de la structure sociale ou des paradigmes dominants. La notion d'agentivité ou agency, qui se traduit parfois par " capacité d'agir ", "capacité d'action " ou "intentionnalité de l'acteur " (Butler, 2006), vise à rendre compte des pratiques par lesquelles les acteurs se jouent du pouvoir ou le remettent en cause. Dans cette optique, la danse n'est pas seulement l'expression 


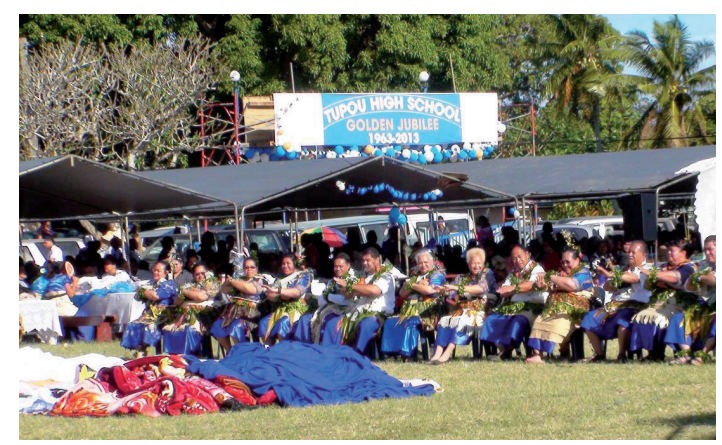

Рното 2. - D’anciens élèves du Tupou High School de Tonga et des diasporas accompagnent leur don d'une danse assise (mäulu'ulu) lors du cinquantenaire de l'institution, juillet 2013, Tonga (C) Condevaux)

mais surtout une pratique du pouvoir (Henry, 2000: 324) ; et on reconnaît aux pratiques corporelles la capacité de déstabiliser les normes sociales (Butler, 2006) tout autant qu’à être déterminées par la structure sociale (Bourdieu, 2000). On voit comment cette perspective peut prolonger et compléter les travaux océanistes qui ont montré, d'une part, la complexité des liens entre représentations et travail du corps et, d'autre part, des rapports de pouvoir politico-religieux. Ces recherches ont particulièrement insisté sur le fait que les rites d'initiations, les processus d'engraissement, les pratiques d'ornementation, etc. permettent de " construire dans le corps la supériorité ou la subordination des individus" (Godelier et Panoff, 1998), mais aussi que le corps, ses constituants ou ses substances sont mobilisés comme symboles de la légitimation des rapports de pouvoir (Godelier et Panoff, 1998 : 23). L'approche de R. Henry, abordant les pratiques corporelles comme pratiques $\mathrm{du}$ pouvoir, nous invite ainsi à un renversement de perspective. L'ethnographie montre effectivement que les performances peuvent être des moyens de subversion vis-à-vis des structures de pouvoir, comme c'est le cas en Polynésie occidentale avec des personnes - généralement proches des chefs appelées "clowns » dans la littérature anglophone (Hereniko, 1994), dont le rôle est non seulement de divertir mais aussi de prévenir de possibles abus de pouvoir (Hereniko, 1994). Ce caractère subversif des performances est tout particulièrement marqué dans les relations de pouvoir héritées de la colonisation (Henry, 2000 ; Mageo, 2008).

$\mathrm{Du}$ point de vue de l'étude des dynamiques sociales et du politique, les pratiques musicales et chorégraphiques sont un objet privilégié pour aborder ce que l'on a nommé les "politiques de l'identité ". La plupart des cas d'étude examinés dans les travaux les plus récents sont des situations dans lesquelles la danse et la musique interviennent dans le cadre d'une présentation ou d'une représentation de l'identité collective. Cet acte de présentation ou de représentation implique pour les acteurs une certaine prise de distance ou une forme de réflexivité vis-à-vis de leur culture, propice à la (re)définition des identités culturelles. On aurait tendance à considérer que ces phénomènes sont propres à la "modernité " océanienne et sont un produit de l'histoire coloniale récente. Or, comme l'ont suggéré A. Kaeppler (2002) ou M. Jolly (1992) notamment, cela ne va pas de soi : il semble que, même dans la période précoloniale, la représentation de la culture dans les échanges entre archipels, par le biais de musiques et de danses, n'était en rien dénuée d'une conscience de la distinction entre un "nous » et des " autres".

Aujourd'hui, les pratiques musicales et chorégraphiques dans le cadre de festivals ou de manifestations commandées par des institutions culturelles ou politiques servent de vecteur à l'affirmation ou à la construction des identités et des appartenances aussi bien à une échelle locale (Teaiwa, 2012), régionale, nationale (Bossen, 2000 ; Sissons, 1999), que transnationale. Ainsi, dans ces festivals entend-on souvent parler de l'identité pan mélanésienne (Kupiainen, 2007), polynesienne, micronesienne ou celle plus englobante encore de Pacific Islander (Condevaux, 2015). Les performances peuvent s'adresser à un public partageant la même langue et les mêmes codes esthétiques que les danseurs - par exemple dans le cadre des festivals nationaux (Teilhet-Fisk, 1996) -, ou à un public d' "étrangers" comme dans le cadre de festivals régionaux ou de performances touristiques (Alexeyeff, 2009 ; Condevaux, 2009 ; Desmond, 1993, 1999 ; Kaeppler, 1977, 1988, $2010 \mathrm{~b}$; Sanger, 1988). Dans tous les cas, elles impliquent, pour ceux qui les produisent, discours et réflexions sur ce qui fait leur «identité ». Les grandes manifestations internationales en Océanie, comme le Festival des Arts du Pacifique - où se croisent et se rencontrent des acteurs aux origines géographiques et culturelles très diverses -, deviennent ainsi des rituels de l'identité (rituals of identity) (Kaeppler, 2002) largement débattus par les membres du groupe et leur société en amont de la manifestation et pendant l'événement même. À des échelles plus restreintes, ce sont par exemple les compétitions de kapa haka en Nouvelle-Zélande ou les performances de musique et de danse lors des cérémonies royales tongiennes, qui sont autant d'occasions d'affirmer une identité liée à un groupe "tribal " ou familial (Nouvelle-Zélande), à un village, à un quartier ou à une île (Tonga). Ainsi, à Nuku'alofa, capitale du Royaume de Tonga, l'identification des habitants aux différents quartiers - très forte et empreinte de rivalités - s'exprime aussi bien dans le port de t-shirts et de tatouages dont le motif peut être le symbole du quartier en question (l'aigle, 'ikale, le lion, laione) que dans la participation à des danses emblématiques qui portent également le nom de ce symbole ('ikale, laione). Quelle que soit l'échelle, des rapports de pouvoir sont fréquemment en jeu dans ces processus d'affirmation 


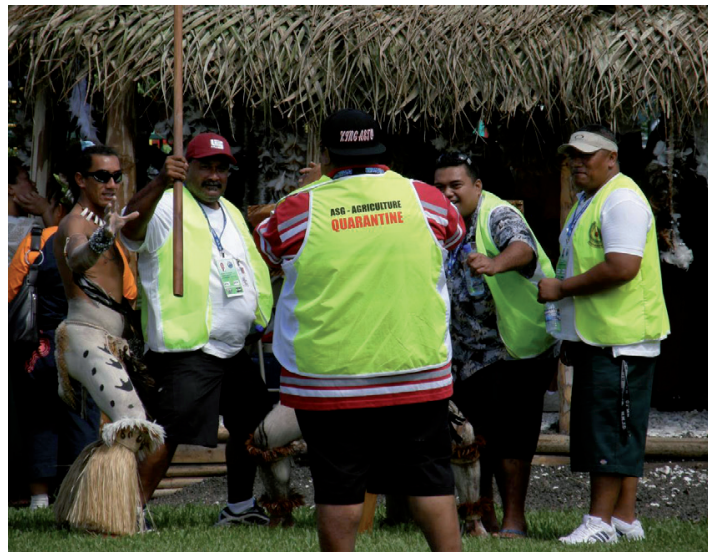

Рното 3. - Danseurs rapa nui en tenue (l'un caché) posant à la demande d'un groupe de bénévoles samoans,

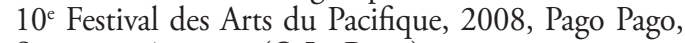
Samoa américaines (C) Le Roux)

identitaire (Butler, 2006 ; Teaiwa, 2012). On peut en rendre compte par l'opposition entre "identité culturelle " et "identité ethnique " proposée par H. Morton Lee (2003). Le premier (cultural identity) est utilisé par l'auteur pour désigner la manière dont les Tongiens se pensent eux-mêmes, alors que l'expression ethnic identity est utilisée pour designer leur réponse aux idéologies et pratiques du multiculturalisme

" in which ethnicity is represented in the public sphere primarily by the outward markers of cultural difference, such as food, music and dance, clothing, and so on." (Morton Lee, 2003 : 5)

À travers les performances culturelles, le corps met en scène des identités sociales à la fois culturelles, ethnicisées et sexuées (Schuft, 2012). Les performances chorégraphiques et musicales sont donc également un lieu privilégié pour l'étude des rapports de genre. En particulier, les performances sont un point d'entrée pour comprendre les tensions et les frictions pouvant naître entre des normes et des catégories sexuées qui seraient exogènes et celles qui seraient endogènes (Alexeyeff, 2008, 2009). Les catégories " endogènes " qui sont (re)produites dans des performances théâtrales ou chorégraphiques, incluent notamment celle des fakafefine, terme tongien qui a divers équivalents selon les langues, notamment fáafafine en samoan, et que l'on peut traduire par "hommes-à-la-manière-des-femmes " (Douaire-Marsaudon, 2008 : 285), une catégorie présente dans de nombreux archipels de l'aire polynésienne (Besnier, 2002; Douaire-Marsaudon, 2008). L'étude de la danse peut également permettre de saisir les transformations des rapports sociaux de sexe, comme le fait A. Tonnaer (2007) dans le cas d'une communauté aborigène du territoire du Nord (Australie). Elle montre, à partir de la comparaison d'une même danse exécutée à plusieurs années d'intervalle, que les transformations de celle-ci sont autant de signes du changement qu'ont connu les rapports sociaux de sexe, sous l'effet notamment du départ des hommes travaillant dans l'industrie du bétail à partir des années 1950.

\section{Un changement de paradigme pour repenser les performances culturelles?}

L'étude des performances a mobilisé divers cadres théoriques. Comme le souligne H. Tateyama dans ce dossier, l'analyse des festivals en Océanie a notamment été inséparable des réflexions sur l'invention des traditions qui ont marqué l'anthropologie océaniste depuis les travaux de R. Keesing et R. Tonkinson (1982) et qui ont alimenté une littérature abondante jusqu'à ces dernières années (Babadzan, 1999, 2009; Friedman, 2002 ; Hanson, 1989; Jolly, 1992; Thomas, 1992). Ces travaux sur les renouveaux culturels, les mouvements politiques et les revendications identitaires en Océanie (Babadzan, 1999, 2009 ; Hanson, 1989; Keesing et Tonkinson, 1982 ; Webster, 1998) n’ont généralement pas pour objet spécifique les pratiques musicales et chorégraphiques. Ils s'y réfèrent souvent, toutefois, pour illustrer les processus d'objectivation ou de réification de la culture à l'œuvre. Les concepts de réification et d'objectivation impliquent l'idée que les "cultures" ou les "traditions " sont définies sur la base d'une sélection délibérée de comportements, de pratiques, de manières de parler ou de vivre, considérés comme emblématiques des groupes concernés. La culture réifiée est de ce fait partielle et limitée. Les performances musicales et chorégraphiques sont au cœur de la définition de cette culture réifiée. Comme le souligne T. van Meijl, par exemple, dans le mouvement politique māori de Nouvelle-Zélande, la culture :

" est généralement définie en insistant sur les aspects expressifs du mode de vie traditionnel, tels que les réunions cérémonielles (hui), la solidarité parentale lors des veillées funéraires (tangihanga), les assemblées religieuses, l'art et les activités matérielles, les chants et les danses. » (1999: 55)

Analysant les processus scéniques à l'œuvre au sein du festival des Arts du Pacifique, A. Babadzan souligne quant à lui que le "travail de réification se matérialise dans la mise en scène des manifestations culturalistes" (2009: 58) qui se présentent notamment par une série :

" [d']items disjoints qui, accolés dans l'inventaire folklorique, sont censés constituer la coutume de chaque ethnie : danses + chants + sculpture + artisanat + architecture + costumes, etc. » (2009: 57) 
À travers ces approches se dessine un premier cadre de lecture possible qui met l'accent sur le caractère fabriqué, (re-)constitué, de ces pratiques et sur les motivations stratégiques qui les sous-tendent. Comme le souligne A. Babadzan :

«On ne peut s'empêcher de faire un parallèle entre ces cérémonies, répliques tropicales des festivals de folklore occidentaux et d'autres 'traditions inventées' européennes de la fin du XIX siècle. " (2009 : 58)

Selon Hobsbawm et Ranger (1983), l'invention des traditions (invention of traditions) est avant tout une manipulation de symboles culturels à des fins politiques, généralement au profit d'une élite éduquée au détriment du peuple. La théorie peut s'appliquer aux sociétés du Pacifique: du fait de leur spécificité, de leur histoire ancienne et récente (dont la colonisation, les reconfigurations politiques et l'émergence de nouvelles élites), elles présentent des inégalités socioéconomiques profondes. C'est le cas, notamment pour ce qui concerne les retombées économiques du développement ou encore des politiques de réparation entreprises en Nouvelle-Zélande qui offrent des compensations financières ou des restitutions de terres dont sont exclus en grande partie les Māori urbains, en particulier ouvriers, au profit d'une élite éduquée qui a su conserver un lien fort avec les territoires ruraux d'origine (Babadzan, 2009 ; van Meijl, 1999 ; Webster, 1998). Ces processus impliquent bien souvent que les traditions "inventées " soient expurgées de toute trace d'influence occidentale (Hanson, 1989; Kempf, 2007). J. Sissons (2005) fait un constat convergent concernant les peuples autochtones en général qui, pour faire valoir leurs droits politiques, doivent bien souvent montrer qu'ils sont radicalement " autres ", répondant ainsi à ce qu'il appelle l'" authenticité oppressive ». Le terme de folklorisation est parfois mobilisé pour désigner ce processus consistant à effacer toutes les traces supposées de contact culturel et impliquant de geler une forme culturelle dans un passé précolonial imaginaire (Balme, 2007 : 119).

L'approche en termes d'invention de la tradition a été largement discutée et il ne s'agit pas ici de rappeler l'ensemble du débat. Le principal point de la critique portait sur le fait que, bien que la plupart des auteurs s'interdisent un jugement sur le caractère "authentique" des "traditions inventées", il n'en demeure pas moins que leurs écrits contenaient un « soupçon d'inauthenticité " à leur égard. Les chercheurs s'inscrivant dans ce cadre interprétatif ont été accusés d'abuser de leur autorité intellectuelle pour distinguer le "vrai » du "faux " et de présenter de manière caricaturale les leaders des mouvements politiques comme de fins stratèges, occidentalisés, capables de "falsifications » ou de "rhétoriques machiavéliques" pour faire valoir leurs intérêts (Wittersheim, 1999: 189-190). Cette critique a généré en retour une méfiance à l'égard des anthropologues, parfois accusés de néo-colonialisme (Tengan et White, 2001).

Un second point essentiel de la critique a porté sur le fait que ces supposées "inventions" n'en étaient bien souvent pas. Par exemple, J. Friedman avance que ce qui a été qualifié d'invention, par Trevor Roper (1983) pour les kilts ou par J. Linnekin (1983 cité par Friedman, 2002) dans le cas d'Hawai'i, relèverait en fait de transformations sociales et s'inscrirait plus dans la continuité que dans la rupture (Friedman, 2002: $223 ; 225 ; 243$ ).

Ces débats concernent pleinement l'étude des pratiques musicales et chorégraphiques. Tout d'abord, la question de l'authenticité a été centrale dans ce champ de recherche. À partir de l'instant où elles sont sorties de leur contexte d'exécution habituel - qu'elles perdent leur caractère " religieux " pour devenir des " emblèmes identitaires " ou qu'elles passent de l'entre soi à une représentation pour les touristes, par exemple, se pose la question du caractère "authentique " de la pratique (Henry, 2000 ; Kempf, 2007 ; Kupiainen, 2007 ; Mackley-Crump, 2016 ; Stevenson, 1999 ; Stillman, 1988). Cependant, l'expression d'invention parait ici tout autant abusive dans les cas traités par Friedman (2002). Dans l'épilogue du numéro de Pacific Studies (1992) déjà évoqué, A. Kaeppler se réjouit que le concept d'invention de la tradition, qu'elle juge peu opérant dans le cadre du Pacifique, ne soit pas utilisé dans la plupart des contributions au numéro. Elle relève, en revanche, les expressions suivantes: "transformation ", "recontextualisation ", "re-création ", " résurrection", " révision" et "négociation ", autant de termes qu'elle juge plus appropriés que celui d'invention dans la mesure où les dynamiques décrites sont souvent loin d'être de " pures inventions». Quelques cas semblent cependant faire exception, comme celui des danses banabans " créées" de toutes pièces pour appuyer des revendications d'indépendance de Banaba (Kempf, 2007), ou celui des danses de Sikaiana, dans les îles Salomon, étudiées par Donner (1992: 71). Ce dernier note que la plupart des danses considérées comme "traditionnelles" à l'époque de son enquête de terrain - dans les années 19801983 - ont été adoptées au début du Xx ${ }^{\mathrm{e}}$ siècle, au contact de Polynésiens. En dépit de cela et bien qu'elles aient connu de profondes évolutions, l'auteur préfère parler de tradition reconstruite plutôt qu' inventée (1992: 79).

En outre, contrairement à ce qui est mis en avant dans le cadre d'analyse de l'invention des traditions, les performances musicales et chorégraphiques - y compris celles utilisées comme emblèmes identitaires des mouvements politiques - ne sont pas systématiquement épurées des influences étrangères et leur pratique peut être un lieu de négociation où se tissent des rela- 
tions de pouvoir antagonistes, que l'on ne peut réduire à la domination d'une élite intellectuelle. Prenons l'exemple des kapa haka (arts "vivants " māori) en Nouvelle-Zélande. La compétition nationale de kapa haka (Te Matatini) tend à renforcer le modèle « ethnique » de la société māori, lequel s'est largement imposé avec la renaissance māori et la politique de réparation déployée sous l'égide du Tribunal de Waitangi ${ }^{1}$. Selon cette logique, les groupes māori sont amenés à se penser selon des liens "ethniques", familiaux ou "tribaux " (iwilhapü/whānau) ${ }^{2}$, qui fondent également la constitution des groupes de danseurs. En outre, les règles de la compétition incitent au maintien de styles, d'accessoires et de costumes jugés " traditionnels " plus qu’à encourager les innovations. Cependant, il existe diverses initiatives qui mettent en avant un visage des autochtones de Nouvelle-Zélande bien loin de celui d'un groupe qui, prisonnier du passé, serait aux prises avec des normes sociales rigides. Une autre compétition, appelée 2 degrees kapa haka super 12, moins célèbre que Te Matatini, en est un bon exemple : tout en présentant des pratiques artistiques qui affirment un ancrage au sein des arts performatifs māori, la réglementation n'encourage en rien la constitution de groupes sur des bases " tribales » ou "familiales » et le champ est laissé ouvert à toutes les innovations, aussi bien dans les costumes et les accessoires utilisés, que dans les chorégraphies. Ces performances n'en sont pas moins entourées de discours affirmant la fierté d'appartenir à un groupe à l'identité culturelle spécifique (que ce soit celle de māori en général, de femme māori ou de tel ou tel iwi ou hāpu). Les pratiques musicales et chorégraphiques māori peuvent donc servir d'appui pour revendiquer des appartenances sociales multiples, qui varient selon les contextes et les enjeux, et qui sont parfois en rupture avec la demande d' "authenticité oppressive " évoquée précédemment. Les discours des acteurs laissent transparaittre des visions de la «culture " ou de la " tradition " qui vont bien au-delà d'une entité figée ou à l'abri des influences extérieures (Condevaux, 2009).

Ce constat s'impose dans d'autres contextes. La vidéo nommée Zorba the Greek Yolngu style filmée en terre d'Arnhem (voir ci-dessous) est un autre exemple de ces tentatives pour rompre avec les stéréotypes et les identités assignées. Ces dernières sont également dénoncées dans le travail d'artistes contemporains qui tentent de trouver une place originale dans les interstices de catégories rigides qui leur sont imposées, que ce soit celles d' "artiste aborigène " ou "artiste māori " par exemple, ou encore, concernant leurs œuvres, celles d'art "traditionnel ", " authentique ", ou " urbain " (Le Roux, 2007 ; Sissons, 2005). L'expression de "réappropriation créative " est mobilisée par B. Glowczewski et J. de Largy-Healy (2014) pour rendre compte de ces productions qui vont à l'encontre des images construites historiquement par l'appropriation occidentale des "patrimoines " océaniens. Ce terme de "réappropriation" est justifié par le fait que les groupes sociaux se ressaisissent de ces objets ou des pratiques jusque-là présentés comme les signes par excellence du primitivisme (le haka, le didgeridoo [yidaki]), définissant euxmêmes leur image ainsi que la manière dont ils veulent vivre et transmettre leurs traditions.

Certains envisagent ce renouvellement des cadres d'analyse comme un changement de paradigme :

"[...] de nombreux travaux de recherche sur les peuples autochtones produits ces dernières années, notamment en France et dans les pays francophones, ont pu montrer un changement de paradigme (Glowczewski et Henry 2007, Gagné et al. 2009, Bosa et Wittersheim 2009). La question actuelle de l'anthropologie ne consiste pas, selon nous, à évaluer l'authenticité des traditions mais à analyser l'efficacité existentielle des nouveaux agencements (Guattari 1992). » (Glowczewski et de Largy-Healy, 2014 : 189)

Cette évolution du cadre théorique implique en outre de nouvelles manières d'aborder les relations de pouvoir, sans nier pour autant l'asymétrie des relations et les inégalités engendrées par l'histoire coloniale, les mouvements politiques et la disparité des accès aux ressources. Bien que les «traditions" soient souvent manipulées par une élite dominante à des fins politiques, les performances musicales et chorégraphiques peuvent également être le vecteur par lequel s'expriment les conflits et les tensions au sein des sociétés, par exemple entre des manières divergentes d'envisager le rapport au passé et à la "tradition ", comme en témoigne l'exemple des compétitions māori. Dans cette perspective, les concepts de " résistance ", "stratégie ", " tactique ", d'"efficacité existentielle» (Glowczewski et de Largy-Healy, 2014 ; Le Roux, 2012) sont amenés à occuper une place importante. Les concepts de "tactiques " et de "stratégies " sont notamment définis par De Certeau (1990). Ce dernier, s’inscrivant dans la continuité de M. Foucault, explore:

«les formes subreptices que prend la créativité dispersée, tactique et bricoleuse des groupes ou des indi-

1. Mis en place en 1975 pour examiner les réclamations déposées par les Māori à l'égard des spoliations de terres ou d'autres ressources dont ils ont été victimes durant la colonisation. Cette politique s'est traduite par des réparations sous forme de compensation financière, de restitution de terres ou autres ressources (quotas de pêche notamment).

2. Les termes utilisés pour désigner ces unités sociales, qui sont souvent traduits par "tribu ", "sous-tribu » et "famille étendue "-ethnic group et tribe en anglais -, portent le nom d'un ancêtre réel ou mythique avec lequel les individus revendiquent un lien de descendance en filiation matrilinéaire ou patrilinéaire. Cela ne signifie cependant pas nécessairement que l'appartenance au groupe est déterminée par une filiation réelle. Voir par exemple à ce sujet Schwimmer (1990). 
vidus pris désormais dans les filets de la "surveillance"."

Ce faisant, il institue une distinction entre tactique et stratégie : la première relève obligatoirement de l'espace de l'autre :

"J'appelle "stratégie" le calcul des rapports de force qui devient possible à partir du moment où un sujet de vouloir et de pouvoir est isolable d'un "environnement" [...] J'appelle au contraire "tactique" un calcul qui ne peut pas compter sur un propre, ni donc sur une frontière qui distingue l'autre

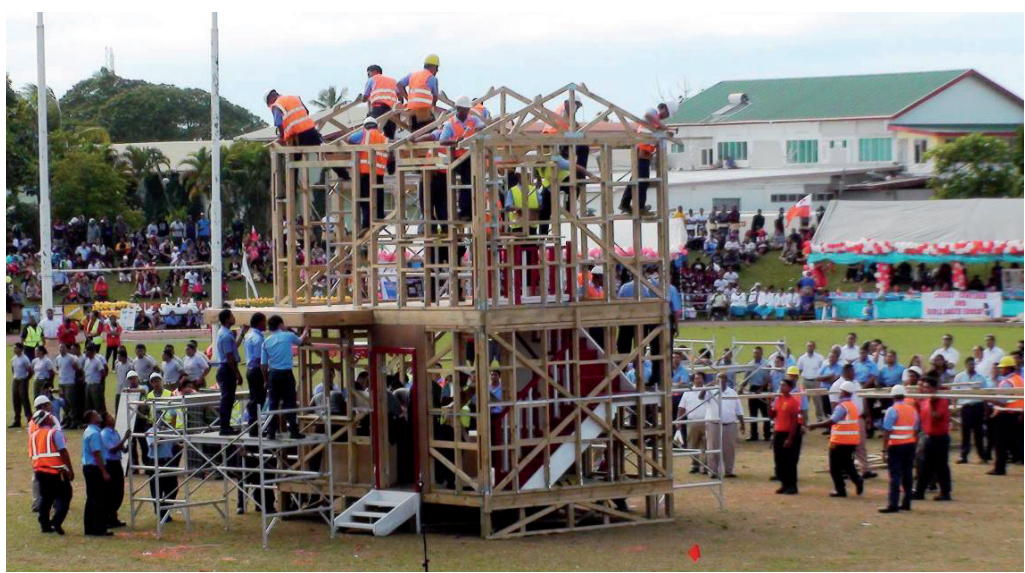

Рното 4. - Démonstration d'une construction de maison, Tonga Institute of Science Technology et Monfort Technical Institute, Jour de l'Éducation, cérémonies du couronnement du roi George Tupou VI, juin 2015 (@ Condevaux) comme une totalité visible.

La tactique n'a pour lieu que celui de l'autre. Elle s'y insinue, fragmentairement, sans le saisir en son entier, sans pouvoir le tenir à distance. » (1990 : XLVI)

L'anthropologue Franca Tamisari (2007), en empruntant la distinction de De Certeau, souligne que les artistes aborigènes agissent comme des agents au positionnement distinct :

«[...] l'art indigène a toujours été et est toujours une tactique performative, une lutte où le protagoniste indigène, contraint de jouer en territoire ennemi, saisit la moindre occasion qui lui est donnée de se manifester et de provoquer une confrontation. " (Tamisari, 2007a : 41)

L'analyse des tactiques déployées pour " résister " au dominant tout en jouant dans son camp rejoint celle développée par certains auteurs des postcolonial ou subaltern studies. Le mimicry de H. Bhabha (1990) illustre bien les "tactiques" employées par des groupes subalternes : le terme désigne une imitation du colonisateur, mais une imitation subversive, parce qu'infidèle.

Dans quelle mesure les contributions publiées dans ce dossier thématique reflètent-elles ces évolutions de cadres théoriques? Permettent-elles de conclure à un " changement de paradigme" ?

\section{Présentation des contributions}

Ce numéro thématique visait à prolonger la réflexion selon trois directions : redéfinir la délimitation d'un champ d'étude non autonome; questionner le rôle transformatif de phénomènes transnationaux contemporains sur les performances culturelles; renouveler et discuter les prismes théoriques permettant de mieux analyser ces dernières. En premier lieu, il s'agissait d'élargir le regard à l'ensemble des pratiques expressives entendues comme des événements dis- tingués du quotidien par divers moyens (décors, costumes, rituels d'accueil, etc.), qui ont une dimension communicative délibérée, qui reposent sur la mobilisation de techniques d'expression variées et qui revêtent la plupart du temps un caractère esthétique. Nous souhaitions en particulier prendre en considération les performances ou les événements expressifs qui sont spécifiquement basés sur des relations entre différentes " équipes " d'acteurs sociaux (au sens de Goffman, 1973), dont l'une joue une représentation à destination de l'autre, ou un "rôle " en fonction de l'autre, et ce faisant affirment ou réitèrent des identités culturelles. Comme indiqué plus haut, les pratiques chorégraphiques - et par extension musicales - occupent une place prépondérante dans cet ensemble. Mais elles sont aussi souvent indissociables d'une esthétique corporelle (Pollock, 1999), ou matérielle, plus large. Les danses máli de la société Huli de Papouasie NouvelleGuinée, qui impliquent un travail d'ornementation corporelle élaboré (Timmer, 2000), sont un exemple parlant en la matière, tout comme les danses de masques de Papouasie NouvelleGuinée (Smidt et Eoe, 1999; Wolffram, 2011). Certaines pratiques conduisent au constat que les productions artistiques et les manifestations culturelles du Pacifique transcendent largement les catégories occidentales (Nero, 1992). Ainsi, la démonstration d'une construction de maison lors des cérémonies marquant le couronnement du roi George Tupou VI à Tonga en 2015 (photo 4) prenait-elle place lors d'une journée dédiée à la démonstration de performances musicales et chorégraphiques. Cette inclusion et le fait que l'ensemble de ces pratiques puissent être rassemblées sous le terme de " faiva »-qui peut désigner un travail, un jeu, une danse, une performance musicale, théâtrale voire cinématographique, qui requiert une certaine habileté -, nous renseignent sur la manière spécifique de concevoir les "performances" dans le contexte tongien. 


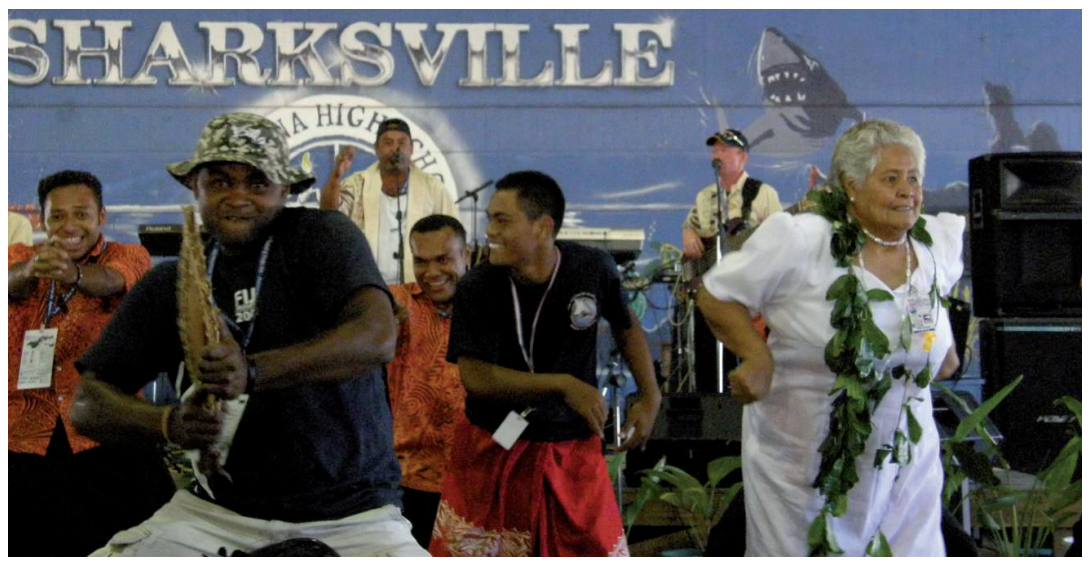

Рното 5. - Intervention dansée spontanée de performers océaniens lors d'un concert de Black Rose à la Jam House, 10 Festival des Arts du Pacifique, 2008, Pago Pago, Samoa américaines (ㄷ Le Roux)

Reflétant cette diversité du champ performatif, les contributions de ce dossier traitent une variété de performances, en termes d'échelle, d'arts et d'artistes mobilisés, de spectateurs, de lieux et de contextes. Il s'agit de spectacles (la Musique de l'eau présentée par T. Dick) ou de prose mise en musique et dansée (F. Aurima-Devatine, E. Castro-Koshy, Moana'ura Tehei'ura et al.) ; de cérémonies ou de festivals au cours desquels des « rituels » se voient attribuer de nouvelles significations (M. Tabani ; H. Tateyama) ; de performance sportive en tant que pratique culturelle (J. Lemarié) ou de festivals artistiques locaux, nationaux ou pan-océaniens (D. Monnerie; C. Graille ; J. Kupiainen).

En deuxième lieu, plusieurs décennies après les premiers travaux sur les festivals (Craig, Kernot et Anderson, 1999), ce dossier cherche à saisir comment les dynamiques sociales contemporaines - notamment le développement des mobilités et des nouvelles technologies de communication - obligent à renouveler l'analyse des performances culturelles. La question des circulations n'est pas tout à fait nouvelle : on peut évoquer à titre d'exemple l'étude de la circulation des drum dances en Polynésie de l'est (Lawrence, 1992 ; Moulin, 1996) et celle de multiples genres de danse en Polynésie occidentale - entre Samoa, Tonga, Fidji, 'Uvea, Tuvalu et Tokelau notamment (Moyle, 1991). Les contacts avec les Européens ont participé à transformer ces circulations, du fait - entre autres facteurs importants - des nouveaux moyens de transport et de communication, mais aussi des nouvelles barrières, qu'ils ont introduites. Les recherches sur les circulations actuelles des musiques et des danses dans le Pacifique (et au-delà) interrogent la manière dont des individus peuvent continuer à "faire société " et à construire un sentiment d'appartenance ou de " connexion " avec leur société d'origine, malgré leur dispersion dans l'espace (Diettrich, Moulin et Webb, 2011 ; Stillman, 1999). La circulation pose également la question des effets de la délocalisation/recontextualisation de la pratique. Les significations et les pratiques du hula hawaiien dépendent par exemple des contextes d'apprentissage, son succès ayant impliqué que celui-ci soit appris - et aujourd'hui également enseigné - par des nonHawaiiens (Stillman, 1999). A. Kaeppler (2010b) montre également comment certaines performances, considérées comme du théâtre dans leur société d'origine, deviennent $\mathrm{du}$ "spectacle " lorsqu'elles sont transposées hors contexte. Par exemple, si les faiva véhiculent un message que le public à Tonga est capable de décoder, leur dimension spectaculaire permet leur intégration sur la scène du Festival des Arts du Pacifique, alors que la capacité du publique à interpréter le sens premier/endogène des paroles et les gestes est perdue (Kaeppler, 2010b : 12).

Les contributions à ce dossier soulèvent cette question de la re-catégorisation des performances ainsi que celle de leur changement de signification, notamment quand elles passent d'un contexte religieux aux usages séculiers et identitaires évoqués précédemment. À partir d'une analyse de l'ancrage multi-scalaire de la performance des Mwerlap du Vanuatu appelée Musiques d'eau, et en prenant en considération plusieurs types de mobilités (nationales et internationales), la contribution de T. Dick à ce volume montre que les imaginaires diasporiques se déploient en référence à une identité localisée dans un lieu-fondateur. Il souligne surtout les effets de la circulation: les changements de rythme (de la vie quotidienne et des contextes de la performance plus que des musiques elles-mêmes), ou la nécessité de re-créer des repères, voire des lieux essentiels au bon déroulement de la performance, lorsque celle-ci se déroule à l'étranger comme lors des tournées australiennes. La question de la recontextualisation des pratiques se pose également dans la contribution de $\mathrm{H}$. Tateyama, qui analyse finement les enjeux et les significations que revêt la démonstration des danses de masques tubuan - produites par les Tolai - lors des festivals nationaux de masque en Papouasie Nouvelle-Guinée. L'auteur montre que la motivation n'est pas ici d'affirmer une identité culturelle particulière mais, pour les Tolai, de réaffirmer leur "supériorité " face aux autres groupes du pays. Les touristes étrangers peuvent passer à côté du sens imputé à la performance, celuici étant directement en lien avec l'histoire et les 
enjeux de pouvoir intercommunautaires, quand on ne leur demande pas tout simplement de quitter le public, certains éléments de la performance devant rester dissimulés aux regards extérieurs. La recontextualisation est ici plus caractérisée par une continuité qu'une rupture. Enfin, les mobilités touristiques et les enjeux économiques qu'elles impliquent participent au redéploiement et à la redéfinition des pratiques. $M$. Tabani montre par exemple comment une même "performance", en l'occurrence les cérémonies John Frum de Tanna, peut passer d'un instrument de résistance à la puissance coloniale au rang d'une attraction majeure pour les tours-opérateurs et les visiteurs étrangers, processus qui s'accompagne par une institutionnalisation de ces fêtes, redéfinies comme "patrimoine".

La question des circulations - des humains, des biens, des pratiques et des images - est inséparable de celle des nouveaux médias. L'importance grandissante de l'usage de ces derniers a été notée par de nombreux auteurs. Par exemple, Diettrich, Moulin et Webb (2011 : 1) indiquent que, lors de la neuvième édition du Festival des Arts du Pacifique en 2004, une rediffusion se faisait pour la première fois en direct sur des écrans géants disposés de part et d'autre de la scène extérieure. Ces auteurs soulignent le caractère élaboré de ce dispositif qui vient confirmer le rôle désormais important de la technologie dans ces événements. B. Glowczewski et J. de Largy-Healy (2014), quant à elles, montrent comment les peuples océaniens mobilisent internet pour valoriser leurs " traditions performatives " et porter leurs revendications politiques à une échelle internationale. L'utilisation des nouveaux moyens de communication est envisagée par ces auteurs comme un moyen de se réapproprier la manière dont les "traditions " sont transmises et exposées et de se ressaisir du contrôle de l'image d'un groupe. Les pratiques chorégraphiques, dont la diffusion se trouve décuplée par l'utilisation d'internet et des réseaux sociaux, peuvent ainsi devenir un instrument pour défaire les préjugés sur la nature supposée figée des "traditions" autochtones : les auteurs donnent l'exemple du clip vidéo Zorba the Greek Yolngu style en terre d'Arnhem. Avec humour, celui-ci défait les préjugés en produisant du burlesque (Glowczewski et de Largy-Healy, 2014 : 197). Bien que le sujet ne soit pas totalement nouveau, l'étude du rôle de ces moyens de communication dans la transformation et la diffusion des pratiques dansées et chantées mérite de plus amples recherches, fondées notamment sur un travail ethnographique. La contribution de J. Kupiainen à ce dossier thématique va en ce sens, analysant la manière dont les identités et l'agentivité culturelles se construisent et se reconfigurent à travers la production et la diffusion d'images visuelles par des groupes culturels des îles Salomon lors du $11^{\mathrm{e}}$ Festival des Arts du Pacifique.

La circulation et la diffusion des pratiques musicales et chorégraphiques posent de fait la question de la propriété intellectuelle. À partir du cas des échanges entre les îles Cook et la Polynésie française, J. Moulin (1996) montrait que, dans les années 1990, la distinction était parfois ténue entre une inspiration artistique légitime et des emprunts vécus comme une dépossession. Comme l'auteur l'annonçait, cette question a gagné en importance ces dernières années du fait que, pour des raisons économiques et politiques, chaque pays est attaché à la particularité de ses danses (Moulin, 1996 : 142). L'article de M. Forsyth et K. Alexeyeff présenté ici aborde cette question, analysant les nouvelles législations sur la propriété des performances culturelles aux îles Cook et leurs origines dans l'anxiété identitaire et les tensions sociopolitiques. Ces "politiques de la créativité et de la tradition ", visant à contrôler les représentations identitaires dansées et les revenus qu'elles peuvent générer, impactent à leur tour la production de pratiques artistiques et leur circulation.

Enfin, en troisième lieu, il s'agit dans ce dossier de discuter les cadres analytiques utilisés actuellement dans l'étude des performances. Les contributions rassemblées ici ne permettent pas de conclure aussi catégoriquement que B. Glowczewski et J. de Largy-Healy (2014, voir citation précédente) à un changement de paradigme. Les articles réunis montrent plutôt l'importance que continue d'avoir le cadre analytique de l'invention des traditions ou, en tout cas, sa discussion tout en montrant le nécessaire dépassement de l'opposition authenticité/inauthenticité. Le terme « invention" semble trop fort dans bien des cas, puisque les pratiques dont il est question ne sont souvent pas des "inventions" pures et simples, mais des transformations d'idées ou de pratiques plus anciennes, et relèvent plus de la continuité que de la rupture (Friedman, 2002 ). Cette continuité culturelle, malgré les ruptures politiques et coloniales qui modifient en profondeur les pratiques et les formes d'autorité ou de contrôle que les acteurs exercent sur celles-ci, est démontrée par certaines contributions de ce dossier. L'article de $\mathrm{D}$. Monnerie souligne la continuité des pratiques et des expressions kanak spécifiques du nord de la Nouvelle-Calédonie (société Arama), grâce à la volonté de les développer, en dépit de la colonisation et en les protégeant d'une exposition aux regards extérieurs. Le texte de M. Tabani montre finalement à la fois des ruptures et des continuités culturelles dans les pratiques cérémonielles étudiées, dépassant ainsi l'opposition entre "indigénisation de la modernité " (Sahlins) et "modernisation de la tradition " (Babadzan). Finalement, que ce soit par la revitalisation d'éléments culturels précoloniaux ou l'emprunt d'éléments exogènes, il s'agit toujours d'établir 


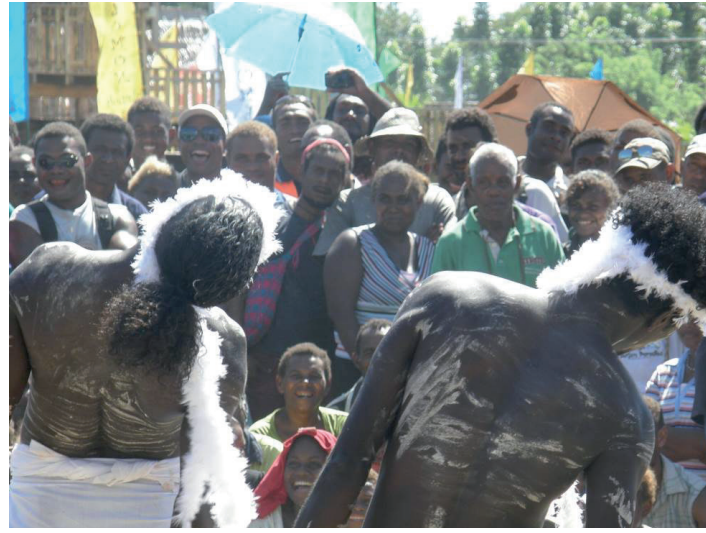

Рното 6. - Performance Zorba, the Greek des Chooky Dancers, $11^{\mathrm{e}}$ Festival des Arts du Pacifique, Honiara, îles Salomon, 2012 (C) Le Roux)

des liens de continuité avec le passé sur une base idéologique, comme le montre $\mathrm{C}$. Graille, à partir de l'étude du festival Melanesia 2000 (NouvelleCalédonie) et des réinterprétations dont il a été l'objet depuis 40 ans. Enfin, J. Lemarié démontre l'usage abusif dont le concept $\mathrm{d}$ '" invention des traditions" a pu faire l'objet à travers l'analyse historique de ce qui s'avère une continuité des pratiques hawaiiennes du surf.

D'autre part, les contributions présentées ici donnent à voir l'agentivité des groupes subalternes, artistes et spectateurs, dont les performances transforment les enjeux sociaux, identitaires, politiques et économiques qui contraignent leur production. Cette agentivité, en tant que volonté d'agir et de définir le monde social et ses catégories, sens et analyses, se donne à voir dans la résistance à la colonisation en Nouvelle-Calédonie (Monnerie) ou dans les productions identitaires entourant la diffusion des images numériques dans les réseaux sociaux (Kupiainen). Elle se donne également à voir à travers la contribution d'Estelle Castro-Koshy, de Flora Aurima-Devatine, Moana'ura Tehei'ura et co-contributeurs à ce dossier autour de Pina'ina'i, événement culturel de Papeete. Leur discussion analyse le sens artistique et sociopolitique de ce spectacle produit à Tahiti à travers les voix et perspectives de multiples catégories de participants : concepteurs, contributeurs, artistes et spectateurs, dont certains chercheurs, revendiquant des catégories d'appartenance variées. Leur discussion rend compte d'une nouvelle forme de relation à la performance dans sa mise en image et en mots, de processus de co-naissance porté par un ensemble hétérogène de personnes réunies pour rendre compte, à un public de lecteurs tahitiens et étrangers, des significations sociales, culturelles et politiques attribuées à cette performance artistique. Cette contribution fait écho aux débats sur la légitimité des chercheurs non autochtones dans le Pacifique, légitimité qui continue à être questionnée par certains (Smith, 1999 ; Teaiwa, 2006), et invite à s'interroger sur les manières de conduire des recherches aujourd'hui dans le Pacifique, de parler "de" ou "pour" les "autres". La place à accorder aux voix subalternes est abordée sous un autre angle dans la contribution de T. Dick. Ce dernier commente de manière explicite la coprésence de différents régimes de savoirs et situe sa propre réflexion au regard de celle de ses interlocuteurs, lesquels mènent leur propre recherche. Il s'interroge également sur les conditions de co-production des connaissances, tout comme D. Monnerie qui fut sollicité dans les années 1990 par des membres du conseil Hoot ma Whaap pour réaliser une "mise par écrit de récits, actes et conceptions de la société Arama, de la culture et de la langue, en particulier à destination des jeunes générations ». Il réalise ainsi un travail ethnographique sollicité par la société Arama, pour la société Arama.

L'ensemble des contributions aborde ces questions par diverses approches théoriques et empiriques et ce, dans de multiples contextes de l'Océanie insulaire. Les contributions s'articulent de la manière suivante. La première contribution, par Hirokuni Tateyama, aborde la performance des Tubuan tolai au festival national du masque de Papouasie Nouvelle-Guinée et la manière dont celle-ci pourrait être interprétée comme un rituel de supériorité vis-à-vis des autres groupes papous. La contribution de Thomas Dick nous emmène dans un village de Vanuatu et au-delà. L'auteur y analyse « la création délibérée des identités diasporiques » à travers les performances de patrimoine culturel par une troupe partant souvent en tournée dans le pays et à l'étranger. De la dimension de "production identitaire" des performances, nous passons à la dimension de "résistance " avec la contribution de Denis Monnerie. Ce dernier aborde les manières dont les gens d'Arama et de Hoot ma Whaap (Nouvelle-Calédonie) résistent à la "patrimonialisation " de leur culture par la créativité sociale et culturelle. En revenant sur les conditions sociologiques et politiques d'émergence du festival Mélanésia 2000, Caroline Graille aborde la " mise en spectacle de l'identité kanak » comme une autre forme de résistance politique. L'analyse des discours portés a posteriori sur l'événement montre qu'il est, avec quarante ans de recul, « le socle d'une idéologie nationaliste ».

Cette contribution est suivie par une discussion à voix multiples, co-écrite par Estelle CastroKoshy, Flora Aurima-Devatine, Moana’ura Tehei' ura et seize co-contributeurs, sur Pina'ina'i. Ce spectacle, innovant à Tahiti, mêle le corporel et le littéraire par la danse et la poésie. Vient ensuite un article de Miranda Forsyth et Kalissa Alexeyeff qui analysent et interrogent les lois et les règlements qui structurent la propriété intellectuelle des performances culturelles aux îles Cook, ainsi que les tensions identitaires qui en résultent ou 


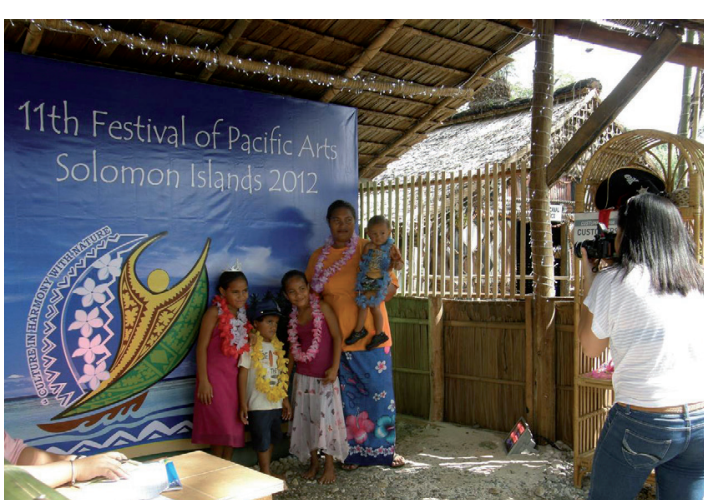

Рното 7. - L'espace média du $11^{\mathrm{e}}$ Festival des Arts du Pacifique, Honiara, îles Salomon, 2012 (C Le Roux)

qui, au contraire, les nourrissent. La contribution de Jari Kupiainen prolonge la réflexion en interrogeant l'utilisation en Océanie des technologies de communication et de la diffusion d'images numériques dans le cadre du Festival des Arts du Pacifique aux îles Solomon, et surtout leur rôle dans la construction d'identité(s) culturelle(s). L'article de Marc Tabani se penche sur la question théorique des continuités ou des changements culturels à travers le cas empirique des cérémonies John Frum à Tanna, Vanuatu. La pertinence d'une approche en termes d'invention ou continuité reste centrale dans le travail sociohistorique de Jérémy Lemarié démontrant la continuité du surf hawaiien en tant que performance culturelle et enjeu identitaire.

De manière transversale, les articles rassemblés dans ce dossier thématique, d'une part, soulignent l'importance des enjeux sociaux, identitaires et politiques dans le renouvellement permanent des performances culturelles en Océanie. Ces dernières ne sauraient être soustraites des relations et luttes politiques et sociales qui les transforment et qu'elles transforment en retour. D'autre part, ces contributions montrent que, si l'usage des technologies récentes de communication et de circulation participent à façonner les performances culturelles contemporaines, ces dernières s'inscrivent aussi dans des continuités historiques et sont parfois un support de la reproduction des relations sociales en contexte de mondialisation. À travers des études de cas empiriques issus de divers territoires océaniens, ce dossier présente une sélection de travaux qui donnent à voir des approches théoriques diverses des transformations et des continuités que présentent les performances culturelles en Océanie.

\section{BIBLIOGRAPHIE}

Alexeyefr Kalissa, 2009. Dancing from the heart. Movement, gender and Cook Islands Globalization, Honolulu, University of Hawaii Press.
—, 2008. Globalizing Drag in the Cook Islands: Friction, Repulsion, and Abjection, The Contemporary Pacific 20(1), pp. 143-61.

Armstrong Alan et Reupena Ngata, 2002. Maori Action Songs: Words and Music, Actions and Instructions, Auckland, Reed.

Babadzan Alain (éd.), 1999. Journal de la Société des Océanistes 109: Les politiques de la tradition. Identités culturelles et identités nationales dans le Pacifique (http://jso.revues.org/ persee-246572).

BABADZAN Alain, 2009. Le spectacle de la culture: globalisation et traditionalismes en Océanie, Paris, L'Harmattan.

Balme Christopher B., 2007. Pacific Performances: theatricality and cross-cultural encounters in the south seas, New-York, Palgrave Macmillan.

Barlow Kathleen, 1992. "Dance when I die !": Context and role in the Clowning of Murik Women, in W. Mitchell (éd.), Clowning as Critical Practice: Performance Humor in the South Pacific, Pittsburgh, University of Pittsburgh Press, pp. 58-87.

Besnier Niko, 2002. Transgenderism, Locality, and the Miss Galaxy beauty pageant in Tonga, American Ethnologist 29 (3), pp. 534-566.

Best Elsdon, [1925] 1976, Games and Pastimes of the Maori, Wellington, A.R. Shearer.

Bнавна Homi, 1994. The location of culture, Londres, Routledge.

Bourdieu Pierre, 2000. Esquisse d'une théorie de la pratique. Précédé de 3 études d'ethnologie kabyle, Paris, Seuil.

Bossen Clauss, 2000. Festival Mania, Tourism and Nation building in Fiji: the case of the Hibiscus Festival, The Contemporary Pacific 12 (1), pp. 123-154.

Burrows Edwin G., 1945. Songs of Uvea and Futuna, Honolulu, Bernice Bishop Museum.

ButLer Judith, 2006. Trouble dans le genre (Gender Trouble). Le féminisme et la subversion de l'identité, Paris, La découverte.

Castellani Jean-Pasquin, 2007. Lidentité corse ou la performance oubliée, in P. Frustier (éd.), Les identités insulaires face au tourisme, La Roche-sur-Yon, Éditions Siloe, pp. 72-77.

Condevaux Aurélie, 2009. Maori Culture on Stage: Authenticity and Identity in Tourist Interactions, Anthropological Forum 19, pp. 143-161.

—, 2010. Touristes, autochtones et fakapale à Tonga : les difficultés d'une rencontre "authentique", Teoros 29 (1), pp. 81-89.

-, 2015. All the same family'? Constructing and Embodying the "Pacific Peoples" Catego- 
ry in New Zealand, in S. Barbour et al. (eds), Diasporas, Cultures of Mobilities, "Race", vol 2: Diaspora, Memory and Intimacy, Montpellier, Presses Universitaires de la Méditerranée, séries PoCoPages, pp. 195-212.

Craig Barry, Bernie Kernot et Christopher AnDERSON (eds), 1999. Art and Performance in Oceania, Honolulu, University of Hawaii Press.

De Certeau Michel, 1990. Linvention du quotidien, tome 1 : L'art de faire, Paris, Gallimard.

Desmond Jane C., 1993. Embodying Difference: Issues in Dance and Cultural Studies, Cultural Critique 26, pp.33-63.

-, 1999. Staging Tourism : Bodies on Display from Hawaiki to Sea World, Londres et Chicago, University of Chicago Press.

Diettrich Brian, Jane Freeman Moulin et Michael Weвв, 2011. Music in Pacific Island Cultures. Experiencing Music, Expressing Culture, New York, Oxford, Oxford University Press.

Donner William W., 1992. It's the Same Old Song but with Different Meaning: Community and Ethnicity in Sikaiana Expressive Culture, Pacific Studies 15(4), pp. 67-81.

Douaire-MARSAUdon Françoise, 2008. La crise des catégorisations relatives à l'identité sexuée. L'exemple du "troisième sexe", in I. Théry et P. Bonnemère (éds), Ce que le genre fait aux personnes, Paris, Éditions de l'École des hautes études en sciences sociales, pp. 277-296.

FAsSin ÉRIC, 2006. Trouble-genre, in J. Butler (éd.), Trouble dans le genre (Gender Trouble). Le féminisme et la subversion de l'identité, Paris, La découverte, pp. 9-15.

Fer Yannick, 2009. Peut-on danser pour Dieu? Le pentecôtisme polynésien entre rigorisme et "réveil culturel", communication donnée au colloque religions populaires et nouveaux syncrétismes.

Friedman Jonathan, 2002. Y-a-t-il un véritable Hawaiien dans la salle ?, in C. Hamelin et É. Wittersheim (éds), La tradition et l'État, Paris, L'Harmattan, pp. 207-245.

Gardiner Wira, 2005. Haka, a Living Tradition, Auckland, Hodder Moa Beckett.

GLOWCZEWsKi Barbara 2007. Introduction. Entre spectacle et politique: les singularités autochtones, in B. Glowczewski et R. Henry (éds), Le défi Indigène. Entre spectacle et politique, Montreuil, Aux lieux d'être, pp. 11-35.

Glowczewski Barbara et Jessica de Largy HeaLY, 2014. Valeurs et réappropriations patrimoniales, des musées à Internet: exemples australiens et polynésiens, in L. Dousset, B. Glowczewski et M. Salaün (eds), Les sciences humaines et sociales dans le Pacifique Sud. Terrains, questions et méthodes, Marseille, Pacific-Credo Publications, Cahiers du Credo, pp. 183-205.

Godelier Maurice et Michel Panoff (éds), 1998. La production du corps, approches anthropologiques et historiques, Amsterdam, éd. des Archives contemporaines.

Goffman Erving, 1973. La mise en scìne de la vie quotidienne, Paris, Éditions de minuit.

Hanson Alan, 1989. The making of the Maori: Culture Invention and its Logic, American Anthropologist 91 (4), pp. 890-902.

Huata Ngamoni, 2000. The Rythm and Life of Poi, Auckland, Harper Collins.

Henry Rosita, 2000. Dancing into Being: the Tjapukai Aboriginal Cultural Park and the Laura Dance Festival, The Australian Journal of Anthropology 11 (3), pp. 322-332.

-, 2007. Conclusion. Réseaux créateurs/créatifs: politiques poétiques d'indigénité, in $\mathrm{B}$. Glowczewski et R. Henry (éds). Le défi Indigène. Entre spectacle et politique, Montreuil, Aux lieux d'être, pp. 301-319.

Hereniko Vilsoni, 1994. Clowning as Political Commentary. Polynesia, then and now, The Contemporary Pacific 6(1), pp. 1-28.

Hobsbawm Eric et Terence Ranger (éds), 1983. The Invention of Tradition, Cambridge, Cambridge University Press.

Jeudy-ballini Monique, 1999. "Dédommager le désir": le prix de l'émotion en NouvelleBretagne (Papouasie-Nouvelle-Guinée), Terrain 32 , pp. 5-20

Jolly Margaret, 1992. Spectres of Inauthenticity, The Contemporary Pacific 4 (1), pp. 49-72.

Kāretu Tìmoti S., 1993. Haka: The Dance of a Noble People, Auckland, Reed.

Kaeppler Adrienne, 1977. Polynesian dance as "Airport Art", in A. Kaeppler, J. van Zile and C. Wolz (eds), Asian and Pacific Dance: Selected Papers from the 1974 CORD Conference. Dance Research Annual, 7, pp. 71-84.

-, 1988. Pacific Festivals and the Promotion of Identity, Politics, and Tourism, in A. Kaeppler et O. Lewin (eds), Come Mek Me Hol'Yu Han': The Impact of Tourism on Traditional Music. Kingston, Jamaica Memory Bank, pp. 121-38.

-, 1992. Epilogue: States of the Arts, Pacific Studies 15 (4), pp. 311-319.

-, 1993. Poetry in Motion: Studies of Tongan Dance. Tongatapu: Vava'u Press.

—, 2002. Festivals of Pacific Arts: Venues for Rituals of Identity, Pacific Arts 25, pp. 5-19. 
—, 2010a. Interpreting Ritual as Performance and Theory, Association for Social Anthropology in Oceania 2010 Distinguished Lecture Oceania 80, pp. 263-273.

-, 2010b. The Beholder's Share: Viewing Music and Dance in a Globalized World, Ethnomusicology 54 (2), pp. 185-201.

KeEsing Roger et Robert Tonkinson (eds), 1982. Mankind 13 (4): Reinventing Traditional Culture: The Politics of Kastom in Island Melanesia (numéro spécial).

KempF Wolfang, 2007. Le premier Festival des Arts du Pacifique Sud revisité. Fabriquer de l'authenticité et le cas des Banabans, in B. Glowczewski et R. Henry (éds), Le défi Indigène. Entre spectacle et politique, Montreuil, Aux lieux d'être, pp. 221-232.

Kirshenblatt-Gimblett Barbara, 1998. Destination Culture: Tourism, Museums and Heritage, Los Angeles and Berkeley, University of California Press.

Konishi Junko, 1999. The relationship between the evaluations of dance performance and the social system in Yap, Micronesia, in B.Craig, B. Kernot et C. Anderson (éds), Art and Perfromance in Oceania, Honolulu, University of Hawaii Press, pp. 7-14.

KräMer Augustin, 1995. The Samoa Islands, vol. II, Honolulu, University of Hawaii Press.

Kupiainen Jari, 2007. Kastom sur scène n'est pas coutume mise en scène. Réflexions sur le premier Festival des Arts et de la Culture Mélanésiens, in B. Glowczewski et R. Henry (éds), Le défi Indigène. Entre spectacle et politique, Montreuil, Aux lieux d'être, pp. 235-255.

Lawrence Helen R., 1992. Is the "Tahitian" Drum Dance really Tahitian? Re-evaluating the Evidence for the Origins of Contemporary Polynesian Drum Dance, Yearbook for Traditional Music, 24, pp. 126-137.

Le Breton David, 2016. La sociologie du corps, Paris, Presses Universitaires de France.

Le Roux Géraldine, 2007. Tactiques urbaines et performances artistiques, in B. Glowczewski et R. Henry (éds), Le défi Indigène. Entre spectacle et politique, Montreuil, Aux lieux d'être, pp. 151-175.

—, 2012. Regards d'artistes sur les processus de patrimonialisation et de commercialisation de la culture aborigène, Journal de la Société des Océanistes 134, pp. 55-64 (http://jso.revues.org/6601).

Linkels Ad et Lucia Linkels, 1999. Hula, Haka, Hoko! An introduction to Polynesian dancing, Tilburg, Mundo Ethnico.
Linnekin J., I983. Defining Tradition: Variations on the Hawaiian Identity, American Ethnologist 10-2, pp. 241-252.

Mackley-Crump Jared, 2016. From Private Performance to the Public Stage: Reconsidering 'Staged Authenticity' and 'Traditional' Performances at the Pasifika Festival, Anthropological Forum 26 (2), pp. 155-176.

Mageo Jeanette, 2008. Zones of ambiguity and identity politics in Samoa, Journal of the Royal Anthropological Institute 14 (1), pp. 61-78.

McLean Mervyn, 1977. An Annotated Bibliography of Oceanic Music and Dance, Wellington, Polynesian Society.

-, 1999. Weavers of Song. Polynesian Music and Dance, Honolulu, University of Hawai'i Press.

Morton Lee Helen, 2003. Tongans overseas. Between two shores, Honolulu, University of Hawai' i Press.

Moulin Jane Freeman, 1996. What's mine is yours? Cultural Borrowing in a Pacific Context, Contemporary Pacific 8 (1), pp. 128-153.

Moyle Richard, 1991. Polynesian Music and Dance, University of Auckland, Centre for Pacific Studies.

Murray David, 2000. Haka Fracas? The dialectics of identity in discussions of a contemporary Maori Dance, Australian Journal of Anthropology 11 (3), pp.345-357.

Nero Karen, 1992. Introduction: Challenging Communications in the Contemporary Pacific, Pacific Studies 15 (4), pp. 1-12.

Petersen Glenn, 1992. Dancing Defiance: the Politics of Pohnpeian Dance Performances, Pacific Studies 15 (4), pp. 13-28.

Pollock Nancy, 1999. Fat is Beautiful: the Body as Art form in the Pacific, in C.Barry, B.Kernot et C. Anderson (éds), Art and Perfromance in Oceania, Honolulu, University of Hawaii Press, pp. 58-63.

PréAud Martin, 2007. Deux mises en scène interculturelles de l'histoire. Du rituel au théâtre chez les Yolngu et les Kija, in B. Glowczewski et R. Henry (éds), Le défi Indigène. Entre spectacle et politique, Montreuil, Aux lieux d'être, pp.103-124.

Sahlins Marshall, 1989 (1985). Des îles dans l'histoire, Paris, Le Seuil.

SAnger Annette, 1988. Blessing or Blight? The Effects of Touristic Dance-Drama on Village Life in Singapadu, Bali in A. Kaeppler et O. Lewin (eds), Come Mek Me Hol'Yu Han': The Impact of Tourism on Traditional Music. Kingston, Jamaica Memory Bank, pp. 89-119. 
Schuft Laura, 2012. Les concours de beauté à Tahiti. La fabrication médiatisée d'appartenances territoriale, ethnique et de genre, Corps 10, pp. 133-142.

Schwimmer Eric, 1990. The Maori hapu: a Generative Model, The Journal of the Polynesian Society 99 (3), pp. 297-317.

Sissons Jeffrey, 1999. Nation and Destination: Creating Cook Islands Identity, Suva, Institute of Pacific Studies, University of the South Pacific.

-, 2005. First Peoples. Indigenous Cultures and Their Futures, Londres, Reaktion Books.

Smidt Dirk et Soroi Marepo Eoe, 1999. A festival to honour the dead and revitalise society: Masks and prestige in a Gamei community (Lower Remu, Papua New Guinea) in B. Craig, B. Kernot et C. Anderson (éds.), Art and Performance in Oceania, Honolulu, University of Hawaii Press, pp. 107-139.

STERn Monika, 2013. Music in Traditional Exchanges in North Vanuatu, Pacific Studies 36, 1-2, pp. 59-76.

Stevenson Karen, 1999. Festivals, Identity and Performance: Tahiti and the $6^{\text {th }}$ Pacific Arts Festival, in B. Craig, B. Kernot et C. Anderson (éds), Art and Performance in Oceania, Honolulu, University of Hawaii Press, pp. 29-36

Stillman Amy Ku'uleialoha, 1988. Images and Realities: Visitors' Responses to Tahitian $\mathrm{Mu}$ sic and Dance in A. Kaeppler et O. Lewin (eds), Come Mek Me Hol'Yu Han': The Impact of Tourism on Traditional Music. Kingston, Jamaica Memory Bank, pp. 145-66.

-, 1999. Globalizing Hula, Yearbook for Traditional Music, 31, pp. 57-66.

TAMisari Franca, 2000. The Meaning of the Steps is in Between: Dancing and the Curse of Compliments, The Australian Journal of Anthropology 11(3), pp. 274-286.

Tcherkezoff Serge, 1997; Culture, nation, société : changements secondaires et bouleversement fondamentaux au Samoa occidental. Vers un modèle pour l'étude des dynamiques identitaires, in S. Tcherkézoff et F. Douaire-Marsaudon, Le Pacifique-sud aujourd'hui. Identités et transformations culturelles, Paris, éditions du CNRS, pp. 309-373

-, 2004a. Tahiti-1768. Jeunes filles en pleurs: la face cachée des premiers contacts et la naissance du mythe occidental (1595-1928), Tahiti, Au vent des îles.

-, 2004b. 'First contacts' in Polynesia: The Samoan Case (1722-1848). Western misunderstanding about sexuality and Divinity, Christchurch,
Canberra, Macmillan Brown Center for Pacific Studies, the Journal of Pacific History.

Tearwa Katerina, 2012. Choreographing Difference: The (Body) Politics of Banaban Dance, The Contemporary Pacific 24 (1), pp. 65-94.

Teilhet-Fisk Jehanne, 1996. The Miss Heilala Beauty Pageant: Where Beauty Is More than Skin Deep, in C. B. Cohen, R. Wilk, et B. Stoeltje (éds), Beauty Queens on the Global Stage: Gender, Contests, and Power, New-York, Routledge, pp. 185-202.

Tengan Ty Kawika K. et Geoffrey M. White, 2001. Disappearing Worlds: Anthropology and Cultural Studies in Hawai' i and the Pacific, The Contemporary Pacific 13 (2), pp. 381-416.

Thomas Nicholas, 1992. The Inversion of Tradition, American Ethnologist 19 (2), pp.213-232.

Timmer Jaap, 2000. Huli Wigmen engage tourists: self-adornment and ethnicity in the Papua New-Guinea Highlands, Pacific Tourism Review 4, pp. 121-135.

Tonnaer Anke, 2007. La danse de l'Avion. Réarticuler les relations de genre au festival de Borroloola, in B. Glowczewskiet R. Henry (éds), Le défi Indigène. Entre spectacle et politique, Montreuil, Aux lieux d'être, pp. 89-101.

Van Meijl Toon, 1999. Fractures culturelles et identités fragmentées: la confrontation avec la culture traditionnelle dans la société maori post-coloniale, Journal de la Société des Océanistes 109, pp. 53-70 (http:// www.persee.fr/doc/jso_0300-953x_1999_ num_109_2_2105).

VAn Zyle Judy, 1988. Tourism and Japanese Bon-Dancing in Hawai'i, in A. Kaeppler et O. Lewin (eds), Come Mek Me Hol'Yu Han': The Impact of Tourism on Traditional Music. Kingston, Jamaica Memory Bank, pp. 75-87.

VIEnne Bernard, 1996. Visages masqués du pays des morts, dans Vanuatu, Océanie, Arts des îles de cendre et de corail, Paris, Éditions de la réunion des Musées Nationaux, Orstom.

Webster Steven, 1998. Patrons of Maori Culture: Power, Theory and Ideology in the Maori Renaissance, Dunedin, University of Otago Press.

Wittersheim Éric, 1999. Les chemins de l'authenticité, L'Homme 151, pp. 181-205.

Wolffram Paul, 2011. 'Singing Spirits and the Dancing Dead': Sonic Geography, music and Ritual Performance in a Melanesian Community, in B. Abels (ed.), Austronesian Soundscapes. Performing Arts in Oceania and Southeast Asia, Amsterdam University Press, pp. 169-191. 